
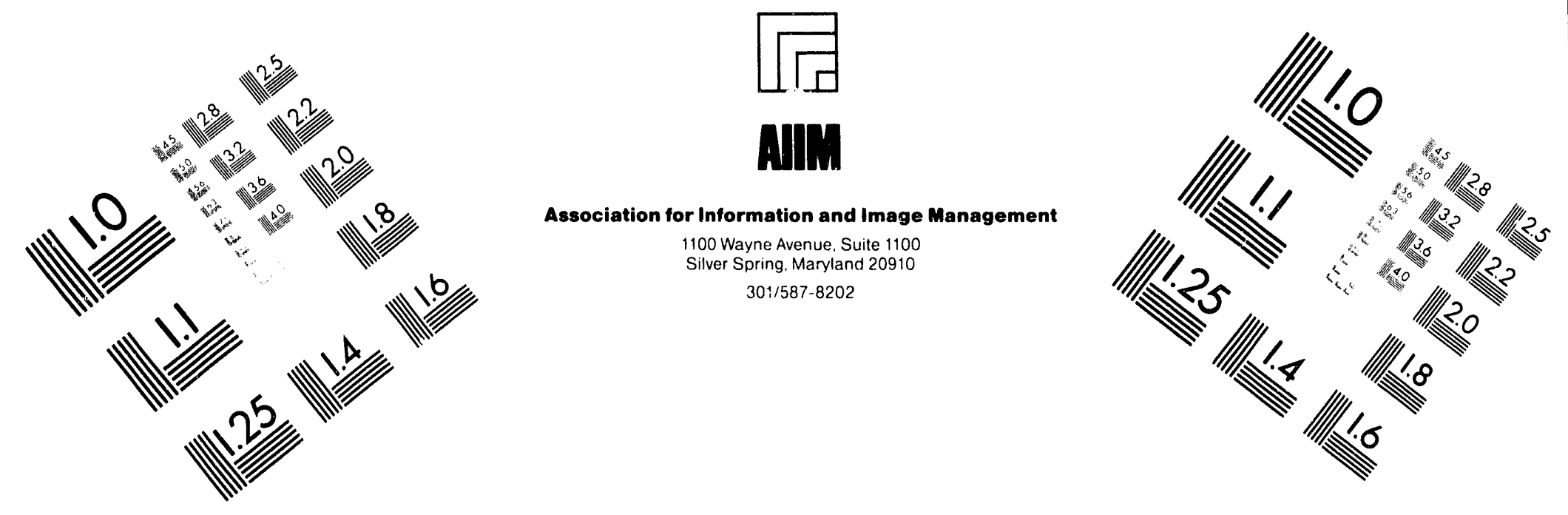

Centimeter

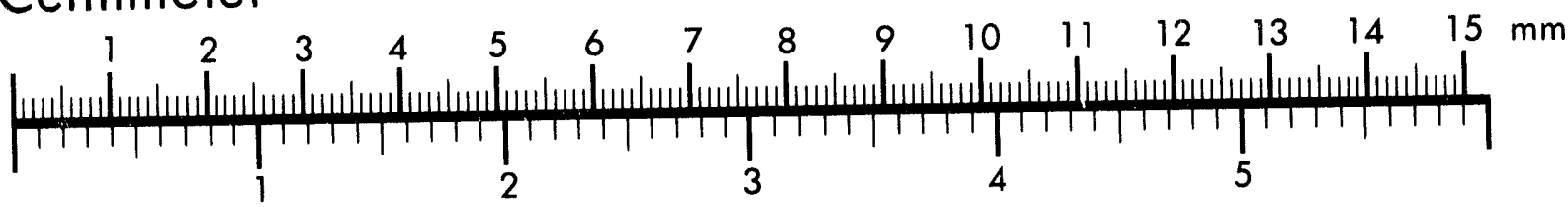
Inches
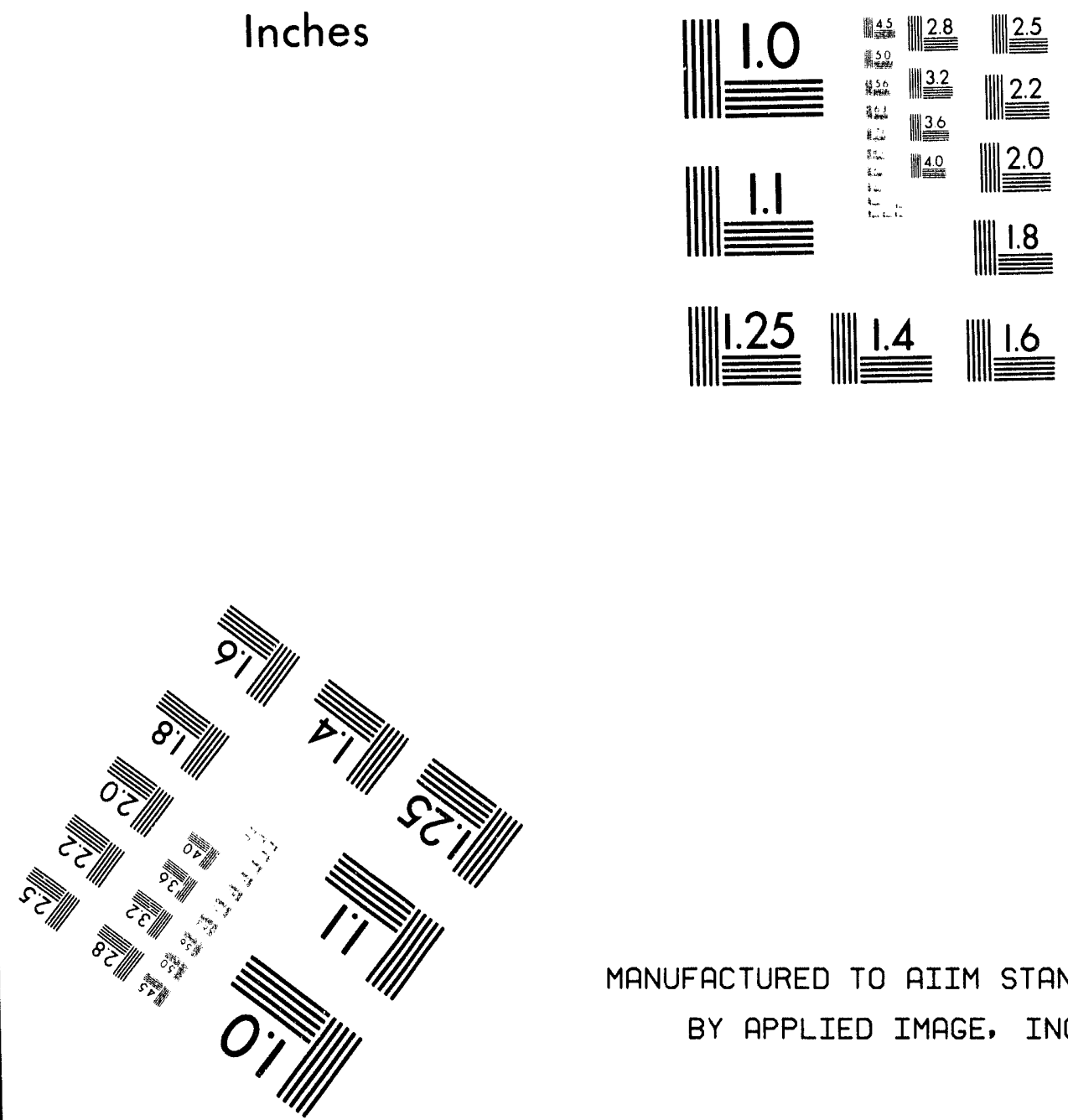

MANUFACTURED TO AIIM STANDARDS

BY APPLIED IMAGE. INC.

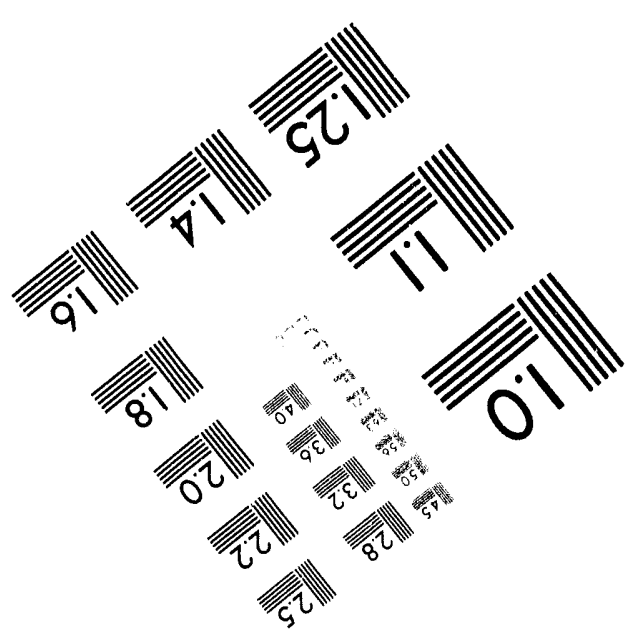



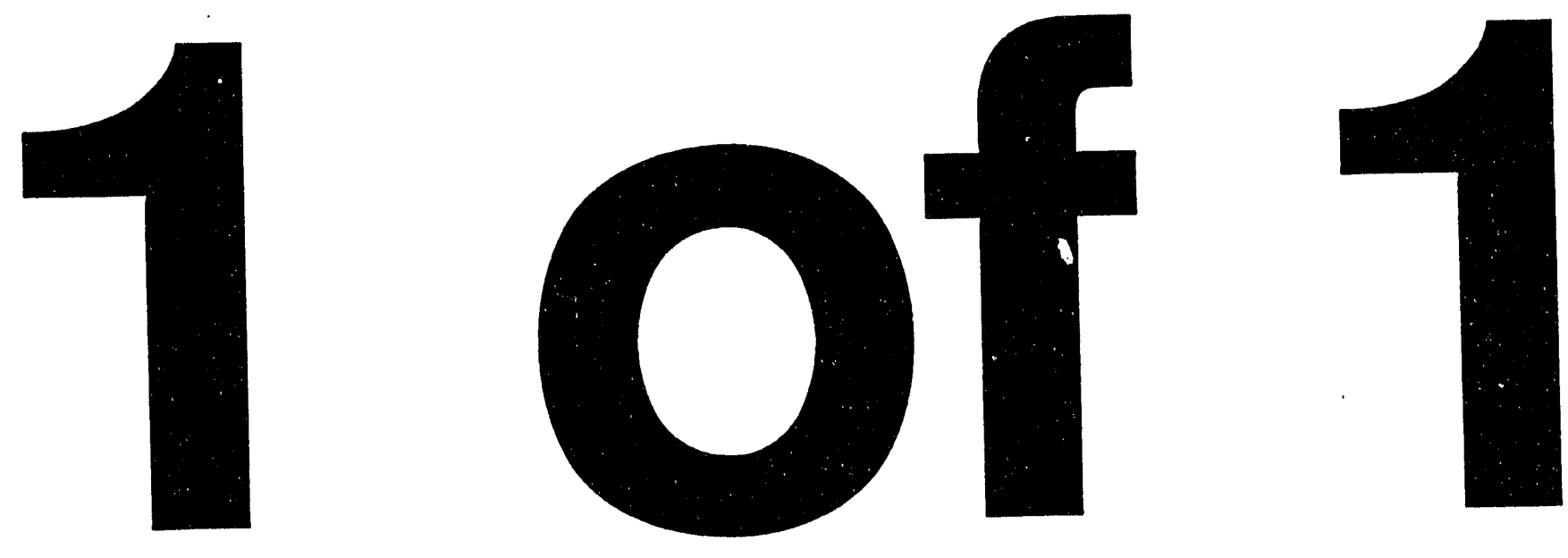
1994 Meeting of the IRIS Specialty Group on Passive Sensors, 14 March 1994

\title{
Panoramic Imaging Perimeter Sensor Design and Modeling
}

\author{
SAND93-2632C \\ Daniel A. Pritchard \\ Sandia National Laboratories \\ Security Technology Department \\ P. O. Box 5800 \\ Albuquerque, NM 87185-0780 \\ 505-844-7444
}

\section{Abstract}

This paper describes the conceptual design and preliminary performance modeling of a 360-degree imaging sensor. This sensor combines automatic perimeter intrusion detection with immediate visual assessment and is intended to be used for fast deployment around fixed or temporary highvalue assets. The sensor requirements, compiled from various government agencies, are summarized. The conceptual design includes longwave infrared and visible linear array technology. An auxiliary millimeterwave sensing technology is also considered for use during periods of infrared and visible obscuration. The infrared detectors proposed for the sensor design are similar to the Standard Advanced Dewar Assembly Types Three A and B (SADA-IIIA/B). An overview of the sensor and processor is highlighted. The infrared performance of this sensor design has been predicted using existing thermal imaging system models and is described in the paper. Future plans for developing a prototype are also presented.

This research was supported by the Defense Nuclear Agency (DNA) under the direction of the tri-services Physical Security Equipment Action Group.

\section{Introduction}

Passive sensors capable of wide area, standoff intrusion detection are gaining increased importance in applications ranging from upgraded fixed perimeter security to rapiddeployment force protection on peacekeeping missions. Adding video and motion detection capabilities to wide area sensors enhances their usefulness and provides the operator immediate visual alarm assessment.

Video motion detection (VMD) systems have been applied to visible imaging and thermal imaging video cameras around perimeters and sensor fields. However, recent evaluations show nuisance alarms are still excessively high [Malone 88, Vigil 92], and the sensors and processors are not designed into an integrated unit. Previous attempts to integrate visible and thermal imaging devices with advanced processing have produced a significant technology base [Pritchard 90, Arlowe 90, Pritchard 92], but a challenging problem remains: to provide robust detection using sophisticated processing in an affordable, reliable package.

Sandia National Laboratories (SNL) has been tasked by DNA to research requirements and technology for, and develop a prototype of, an Advanced Exterior Sensor $(\boldsymbol{A E S})$ for intrusion detection and assessment. The $A E S$ is to be used in 
ground-based security applications for standoff intrusion detection of humans and vehicles. It will be rapidly deployable and simple to set up and operate. The $\boldsymbol{A E S}$ shall be effective in degraded weather conditions and day and night operations.

\section{Review of Requirements for Stand-Off Intrusion Detection}

Currently, an exterior security sensor with the following capabilities does not exist:

1. Capable of wide area coverage (hundreds of meters for humans and vehicles).

2. Automatic detection, tracking, and classification of multiple targets,

3. Low life-cycle cost.

4. Passive (low electromagnetic signature),

5. Limited mechanical moving parts.

6. Capable of 24-hour operation in varying environmental and climatic conditions,

7. Capable of detecting a wide range of penetration scenarios.

8. High mean time between failure (MTBF) and low mean time to repair (MTTR).

9. High probability of detection $(\mathrm{Pd})$.

10. Capable of sectorized assessment while maintaining detection in the remaining areas of interest.

11. Low nuisance alarm rate (NAR) by discriminating humans and vehicles from nuisance sources normally encountered in an exterior environment.

\section{Detection and Tracking Requirements}

The current human and vehicle detection requirements are summarized in Table 1 . In addition, targets moving as slowly as 0.25 meter per second $(0.1 \mathrm{~m} / \mathrm{sec}$ desired) should be detected.
Table 1. Detection range requirements.

\begin{tabular}{|c|c|c|c||}
\hline Target & Conditions & $\begin{array}{c}\text { Range } \\
\text { (reg'd) }\end{array}$ & $\begin{array}{c}\text { Range } \\
\text { (desired) }\end{array}$ \\
\hline $\begin{array}{c}\text { Upright } \\
\text { human } \\
\text { walk/run } \\
0.6 \times 1.65 \mathrm{~m} \\
1.0 \mathrm{~m}^{2}\end{array}$ & $\begin{array}{c}\text { Clear, } \\
\text { good } \\
\text { visibility } \\
\text { Light rain, } \\
\text { humid }\end{array}$ & $500 \mathrm{~m}$ & $750 \mathrm{~m}$ \\
\hline $\begin{array}{c}\text { Crawling } \\
\text { human } \\
\text { head-on } \\
0.5 \times 0.3 \mathrm{~m} \\
0.15 \mathrm{~m} 2\end{array}$ & $\begin{array}{c}\text { Clear, } \\
\text { good } \\
\text { visibility } \\
\text { Light rain, } \\
\text { humid }\end{array}$ & $250 \mathrm{~m}$ & $525 \mathrm{~m}$ \\
\hline $\begin{array}{c}\text { Truck/van } \\
1.5 \times 1.5 \mathrm{~m} \\
2.3 \mathrm{~m}^{2}\end{array}$ & $\begin{array}{c}\text { Clear, } \\
\text { good }\end{array}$ & $1000 \mathrm{~m}$ & $375 \mathrm{~m}$ \\
visibility & & $300 \mathrm{~m}$ \\
\hline
\end{tabular}

Nuisance alarms should be reduced by distinguishing humans and vehicles from other moving objects (birds, trees, debris). As a goal, $A E S$ is to classify at least $90 \%$ of potential threats vs. non-threats. Detection is to continue while assessing alarms from other locations.

$A E S$ is to accomplish these detection requirements using a continuous $360^{\circ}$ azimuth scanning sensor. The elevation angle can vary to handle uneven terrain. Given the above ranges and coverage, Figure 1 shows a proposed $\boldsymbol{A E S}$ application with sensor modules at each corner of a one square kilometer protected area covering an excess of one square mile.

\section{Environmental Requirements}

It is desired to operate the $\boldsymbol{A E S}$ in a variety of environmental conditions ranging from day to night, hot to cold, and dry to humid and rainy or snowy. The required operating range is $-20 /+120^{\circ} \mathrm{F}\left(-29 /+49^{\circ} \mathrm{C}\right)$, with a desired range of $-40 /+140^{\circ} \mathrm{F}\left(-40 /+60^{\circ} \mathrm{C}\right)$. 


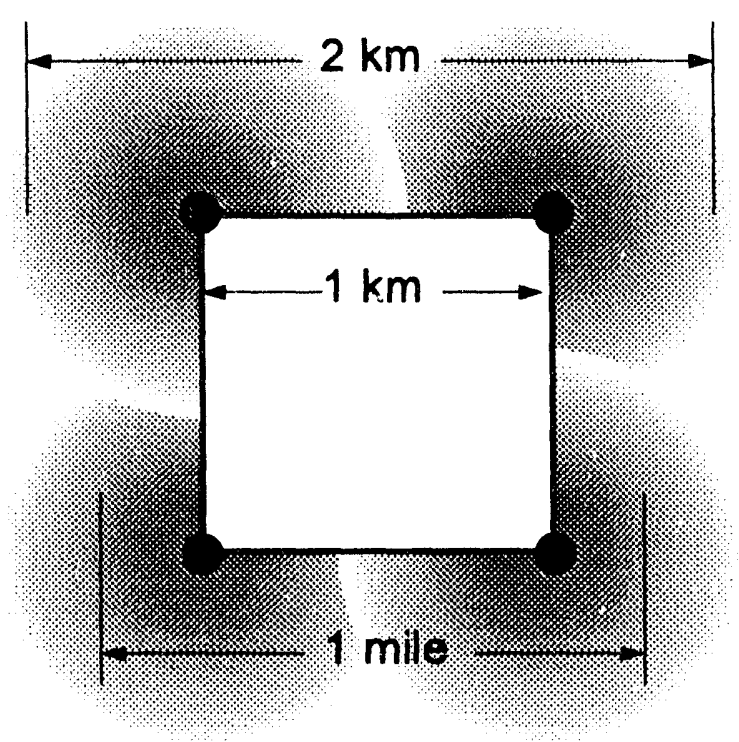

Figure 1. Proposed $A E S$ Application

\section{Other Features}

Alarms and imagery will be remotely displayed for operator evaluation. The $\boldsymbol{A E S}$ shall include a method to compensate for some sensor motion without stabilization. Battery operation of the remote sensor head, with solar charging, is highly desirable. The data processor module, which is remotely located from the sensor module, can be AC powered. Nominally, the system should easily survive shock and off-road vehicle vibrations and have minimum moving parts for 6000 hours or more reliability. Data links and built-in circuits and software should include self-test and diagnostics (tamper, low battery, system fail, etc.).

\section{Advanced Exterior Sensor Conceptual Design}

The goal of the $A E S$ project is to seek out recent advances that may provide the technology to develop an exterior intrusion detection sensor to meet or exceed the above capabilities. Such a sensor, using advanced image processing hardware and algorithms, may be used to intelligently process data derived from detectors and arrays operating in radio frequency ( $R F$ ), infrared and visible wavebands. A block diagram of the $A E S$ is shown in Figure 2.

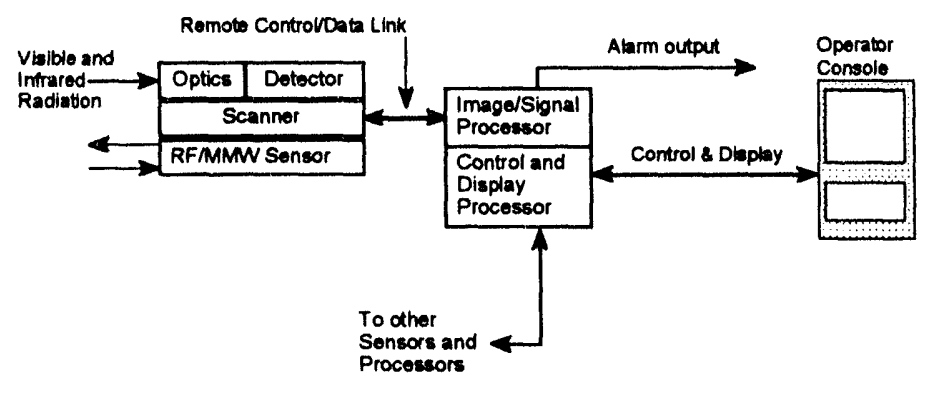

Figure 2. Simplified AES Block Diagram

\section{Panoramic Imaging Remote Sensor Module (RSM)}

\section{Infrared Sensor}

A second-generation, common-module type linear infrared array detector-dewar-cryocooler is the primary sensing device in the $\boldsymbol{A E S}$ infrared sensor module. Two detectors have been considered for $\boldsymbol{A E S}$. These are the Texas Instruments $240 \times 2$ verticaliyintegrated photodiode (VIP) array and the SOFRADIR 240x4 time-delay and integration (TDI) array. The optical system for use with either detector array has not yet been completely defined.

Both detectors under consideration are photovoltaic mercury-cadmium-telluride, operate in approximately the 8-10 micron waveband, and are cryogenically cooled. The TI $240 \times 2$ VIP detector assembly includes a linear-drive cryocooler and is representative of detectors that will be manufactured in modest quantities in the near future. Very good sensitivity has been reported by Texas Instruments [Beck 92]. The SOFRADIR 240x4 TDI detector also requires cryogenic cooling, but the currently 
recommended cryocooler has a cooling capacity of one watt. This is significant when destined for battery-powered applications, because the average power required to drive a one-watt cooler is about 30 watts.

A third detector option, lead-selenide $(\mathrm{PbSe})$, has also been considered. Preliminary modeling predicts the NET to be about $0.6{ }^{\circ} \mathrm{C}$, which is considered high for this application. $\mathrm{PbSe}$ is still being investigated.

\section{Visible Sensor}

Visible-waveband imaging has significant advantages in daytime operation. It can be used to effectively supplement the limitations of an infrared sensor during periods of low thermal contrast in warm background, daytime operation [Arlowe 92, Pritchard 93] Advanced, high-sensitivity, time-delay-andintegration, visible waveband line scanners that operate at high scan rates have adequate sensitivity and can operate simultaneously with the infrared detectors.

\section{Millimeterwave Sensor}

Electromagnetic waves at millimeter wave (MMW) radio frequencies (RF) have the ability to detect humans and vehicles and provide substantially improved atmospheric penetration over the visible and infrared wavebands. For this reason, RF systems have been considered as a potentially important component of $\boldsymbol{A E S}$.

Two types of RF systems have been chosen for potential application to $\boldsymbol{A E S}$. These are pulse-Doppler radar and pulse radar with area moving target indication (AMTI). Both these concepts have become affordable due to advances in integrated circuits. Either approach could operate at microwave or millimeter wave frequencies (up to $94 \mathrm{GHz}$ ). Neither type of radar is appropriate as a sole sensor for AES, since high-resolution imaging for alarm assessment is not provided. However, either type would complement an infrared and/or a visible wavelength sensor. In particular, Doppler radar provides excellent slow motion detection of targets moving directly toward the sensor compared to passive IR and visible video motion detectors that best detect perpendicular motion.

\section{Data Processor Module (DPM)}

\section{Proposed Configuration}

The proposed DPM consists of:

1. Industry-standard VMEbus backplane and industrial quality enclosure.

2. Custom sensor interface board with auxiliary digital inputs and outputs and an image compression circuit.

3. High-speed pipelined pixel processor,

4. Systolic array processor for high-speed filtering operations.

5. Array of high-speed numeric processors for special algorithm operations.

6. General purpose processor for system resource management and overall control.

A moving-target-indicator (MTI) algorithm technique is a good candidate to satisfy the detection and false alarm requirements for the $A E S$. The technique of interest uses Gabor filters to assist in providing benefits such as noise cancellation, immunity to illumination changes, and object motion direction selectivity [Munno 93]. The property of direction selectivity is of special interest for the $\boldsymbol{A E S}$. If the object's motion direction is known, the image could be processed with a Gabor scalar motion filter sensitive in that particular direction. Noise and clutter (and motion) in other directions are attenuated, resulting in a net increase in the target signal with respect to the background noise. 


\section{Processing Requirements}

To process the 6-12 Mbytes per second (or more) of data expected from the $\boldsymbol{A E S}$ sensor head, motion detection algorithms which meet the $A E S$ performance requirements may require 2-10 Giga-OPS (billion operations per second) or more of processing power. This performance may be readily available in moderate-cost, pipelined, systolic, and multi-processor computers in the next few years.

A necessary criterion for the new motion detection algorithms is that the processing can be efficiently pipelined or divided between multiple processors without incurring a heavy overhead penalty. The detection technique considered for the $\boldsymbol{A E S}$ meets this criterion. Another short-term requirement imposed on the algorithms is that operations be computable in integer format without adversely affecting desired results. This requirement is common for high-speed signal processing systems and the impact of this constraint is being investigated.

The simultaneous processing of both visible and IR wavebands is not currently planned; sensor inputs to the data processor will be switched, depending on conditions. Fusion of imagery will be consider for future work.

\section{Display and Control Module}

Although not yet designed or specified, the $A E S$ display and control module will likely consist of an entirely off-the-shelf product from a manufacturer of related products, industrial-controls, or ruggedized computer displays. Flat-panel and video displays are continuously being improved by the small TV and laptop computer industry, and suitable products are expected in the next few years. The display and control module will have the capability of connecting to and controlling multiple sensor modules, while also displaying imagery from the sensor modules. Remote display of the sensor head imagery may require some form of compression and decompression, but rapid progress is being made in these areas.

\section{Infrared Imaging Sensor Performance Modeling}

Two infrared imaging system performance models were used to evaluate the infrared sensor of the $\boldsymbol{A E S}$, FLIR92 and IRDOC.

\section{FLIR92 Performance Model}

FLIR92 is a desktop computer model that predicts basic performance figures-of-merit for thermal imaging systems [US Army 93]. From basic system-level parameters, the model calculates modulation transfer function (MTF), noise equivalent temperature difference (NET), minimum resolvable temperature difference (MRT), and minimum detectable temperature difference (MDT).

FLIR92 was used to predict whether the AES conceptual design achieves the required MTF, system noise, and MRT determined necessary to perform mission-specific detection and tracking tasks. FLIR92 was used with both detector options. Table 2 presents the variables and results achieved using FLIR92 for cases of the TI 240x2 VIP and the SOFRADIR $240 \times 4$ detector configurations. The last two rows of the table show that NEDTs of 0.11 and 0.06 are predicted for the $A E S$ in this configuration, with corresponding apertures of $33 \mathrm{~mm}$ (1.3 inches) and $38 \mathrm{~mm}$ (1.5 inches). The computed 2DMRT curves are presented in Figure 3.

A standing person at $500 \mathrm{~m}$ subtends 1.2 milliradians (mr) in the horizontal direction. The Figure 3 2D-MRT curves show sensitivities on the order of $0.6^{\circ} \mathrm{C}$ and $0.3^{\circ} \mathrm{C}$ for the TI and SOFRADIR arrays, respectively, at a spatial frequency of $1.2 \mathrm{cycles} / \mathrm{mr}$. 
1994 Meeting of the IRIS Specialty Group on Passive Sensors, 14 March 1994

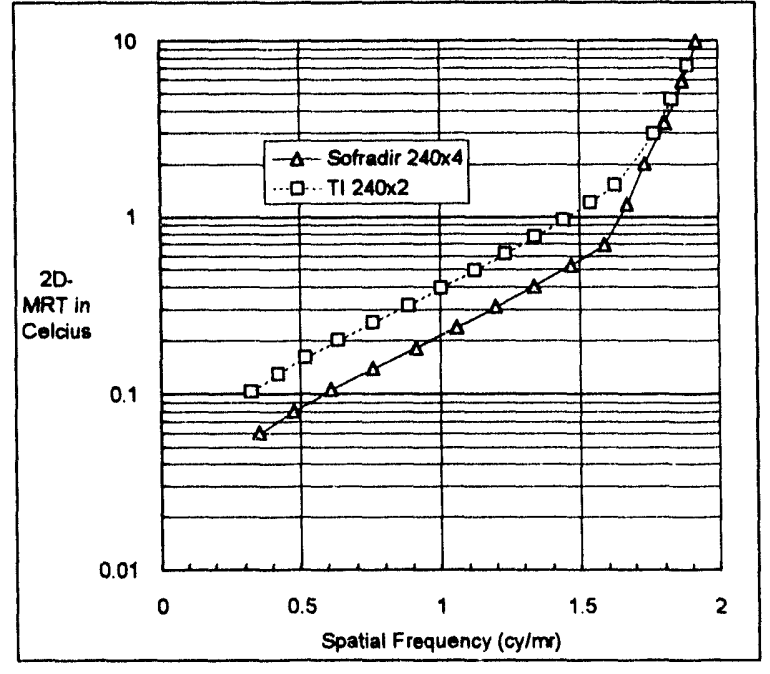

Figure 3. Computed $A E S$ MRT Curves

Note, however, that the 2D-MRT is the geometric-mean of the horizontal and vertical MRT calculations. The horizontalonly MRT is significantly better, at approximately $0.22^{\circ} \mathrm{C}$ and $0.13^{\circ} \mathrm{C}$ for the TI and SOFRADIR arrays, respectively, again at 1.2 cycles/milliradian. It is expected that this general level of performance would be adequate to accomplish the detection task.

\section{IRDOC Performance Model}

The InfraRed Design Optimization Code (IRDOC), developed under the Naval Research Laboratory (NRL), is an optimization code for the design of infrared systems. The model predicts the signal-tonoise (SNR) ratio, including clutter, by taking a number of system parameters and constraints and ranges on the variables from the user and produces a set of design parameters within their allowed ranges that maximizes the SNR [Karins 92].
Table 2. FLIR92 system model parameters for proposed AES sensor.

\begin{tabular}{|l|l|l|}
\hline $\begin{array}{l}\text { Detector/Design } \\
\text { Parameter }\end{array}$ & $\begin{array}{l}\text { TI 240 } \\
\mathbf{2} \text { VIP }\end{array}$ & $\begin{array}{l}\text { Sofradir } \\
\mathbf{2 4 0} \times \mathbf{4}\end{array}$ \\
\hline System F/number & 2.3 & 2.0 \\
\hline Cut-on, cutoff $(\mu \mathrm{m})$ & $7.7-10.3$ & $7.7-10.3$ \\
\hline Detector size horizontal & $22 \mu \mathrm{m}$ & $24.8 \mu \mathrm{m}$ \\
\hline Detector size vert. & $32 \mu \mathrm{m}$ & $27.9 \mu \mathrm{m}$ \\
\hline Detector IFOV - horiz. & $0.29 \mathrm{mr}$ & $0.33 \mathrm{mr}$ \\
\hline Detector IFOV - vert. & $0.42 \mathrm{mr}$ & $0.37 \mathrm{mr}$ \\
\hline $\begin{array}{l}\text { Peak specific detectivity } \\
\text { (D*) }\end{array}$ & $\begin{array}{l}7.6 \mathrm{E} 10 \\
(\mathrm{est} .)\end{array}$ & $\begin{array}{l}7.6 \mathrm{E} 10 \\
(\mathrm{est} .)\end{array}$ \\
\hline Effective focal length & $76.2 \mathrm{~mm}$ & $76.2 \mathrm{~mm}$ \\
\hline System FOV - vertical & $4.6^{\circ}$ & $5.0^{\circ}$ \\
\hline Overall optical efficiency & 0.7 & 0.7 \\
\hline Aspect ratio & 78.58 & 71.44 \\
\hline Samples per HIFOV & 1.5 & 1.7 \\
\hline Samples per VIFOV & 1.26 & 1.0 \\
\hline Blur spot diameter & $0.66 \mathrm{mr}$ & $0.57 \mathrm{mr}$ \\
\hline Samples/mr - horizontal & 5.2 & 5.2 \\
\hline Samples/mr - vertical & 3.0 & 2.7 \\
\hline Scanner rate & $1 \mathrm{rev} / \mathrm{sec}$ & $1 \mathrm{rev} / \mathrm{sec}$ \\
\hline HIFOV's per revolution & 21845 & 19275 \\
\hline Samples per revolution & 32768 & 32768 \\
\hline $\begin{array}{l}\text { ACQUIRE Pd 0.6xl.65 m } \\
\text { man, 0.5 }{ }^{\circ} \mathrm{C}, \text { 500 m, } 2 \mathrm{cy}\end{array}$ & 0.56 & 0.77 \\
\hline Equiv. noise bandwidth & $20 \mathrm{KHz}$ & $20 \mathrm{KHz}$ \\
\hline Dwell time (microseconds) & 25.0 & 25.0 \\
\hline Effective aperture dia. & $33 \mathrm{~mm}$ & $38 \mathrm{~mm}$ \\
\hline Predicted NETD (white) & $0.11{ }^{\circ} \mathrm{C}$ & $0.06{ }^{\circ} \mathrm{C}$ \\
\hline
\end{tabular}


A slightly different set of $\boldsymbol{A E S}$ design parameters, listed in Table 3 , was provided to NRL for processing with IRDOC. The parameters gave results that were comparable to those achieved with FLIR92. Several other parameters were used in the analysis but have been omitted here for brevity.

It should be noted that in this case the target is a $1.5 \mathrm{~m} \mathrm{x} 1.5 \mathrm{~m}$ vehicle with a target-tobackground difference of $2.0 \% \mathrm{C}$. This target was considered for this analysis because it subtends a smaller angle than a standing person at shorter range.

The results of the IRDOC analysis for this configuration show an instantaneous field-ofview (IFOV) of $0.26 \times 0.29$ (horizontal and vertical), with a 4.0 degree total vertical FOV. The computed contrast signal-tonoise ratio (SNR) is 8.5 for the high clutter case. The model was rerun with the clutter parameters set to low; a contrast SNR of 28.7 was predicted. It is estimated that this SNR is sufficient to meet the performance criteria of the $\boldsymbol{A E S}$.

\section{Summary and Future Efforts}

The current $\boldsymbol{A E S}$ design includes an infrared linear array scanner, a visible linear array scanner, with or without a pulse Doppler or area MTI radar adjunct. The infrared detector array modeling shows acceptable resolution and noise levels from the proposed design. Still, contrast sensitivity in both the IR and visible wavebands must be maximized for good performance in poor weather conditions. If a facility or rapid deployment force is allowed to use active RF sensors, an $\boldsymbol{A E S}$ with an active radar using either Doppler or AMTI processing can likely achieve higher performance, particularly in degraded conditions.
Table 3. IRDOC system model parameters for proposed AES sensor.

\begin{tabular}{|l|l|}
\hline Parameter & Value \\
\hline Target shape & extended \\
\hline Area & $2.3 \mathrm{~m}^{2}$ \\
\hline Optics & unobscured \\
\hline Aperture & $4.8 \mathrm{~cm}$ \\
\hline F/\# & 2.0 \\
\hline Transmission & 0.6 \\
\hline Distance & $1000 \mathrm{~m}$ \\
\hline Temp. & $302 \mathrm{~K}$ \\
\hline Scan speed & $6283 \mathrm{rad} / \mathrm{sec}$ \\
\hline Detector & PV-MCT \\
\hline Cut-on & $7.7 \mu \mathrm{m}$ \\
\hline Cut-off & $10.3 \mu \mathrm{m}$ \\
\hline Pixel width & $25.0 \mu \mathrm{m}$ \\
\hline Pixel height & $28.0 \mu \mathrm{m}$ \\
\hline Pixel pitch & $28.0 \mu \mathrm{m}$ \\
\hline TDI columns & 1 \\
\hline Array temp & $77 \mathrm{~K}$ \\
\hline Cold shield $\eta$ & 0.98 \\
\hline Integ time & $25 \mu \mathrm{sec}$ \\
\hline Reset time & $3.0 \mu \mathrm{sec}$ \\
\hline Atmosphere & $\mathrm{US} \mathrm{Standard}$ \\
\hline Background & $300 \mathrm{~K}$ \\
\hline Clutter & high \\
\hline
\end{tabular}

Data processing requirements play a major role in the AES system design. Algorithms that can detect moving objects reliably in low signal-to-noise conditions must be used. 
Further research must be done to understand the capabilities of these algorithms for detection of humans and vehicles in varied and dynamic clutter environments, since this is a largely unexplored area.

Plans include evaluating the computational resources needed to process the 6-12 Mbytes per second (or more) of data expected from the $A E S$. Is may be possible to process this data using commercially available, dedicated pipelined and systolic image processing hardware for the low-precision integer computations, and high-speed multiprocessor computers for the high-precision operations.

\section{References}

Arlowe-90 "The Mobile Intrusion Detection and Assessment System (MIDAS)," H. D. Arlowe, D. Coleman, SAND89-3016C, Proc. IEEE Carnahan Conference on Security Technology, 1990.

Arlowe-92 "Environmental Sensitivity of Thermal Intrusion Detection," H. D. Arlowe, R. C. Dykhuizen, Proc. 33rd Annual Meeting of the Institute for Nuclear Materials Management, 1992.

Beck-92 "240x1 LWIR Vertically Integrated Photodiode Focal Plane Array," J. Beck, et al., Proc. IRIS Specialty Group on Infra-red Detectors, ERIM, Ann Arbor MI, March 1992.

Karins-92 "IRDOC, An IR Sensor Design Optimization Program, Proc. IRIS Specialty Group on Passive Sensors, ERIM, Ann Arbor, MI, March 1992.

Malone-88 "Initial Evaluation and Testing of Adpro and DAVID Video Motion Detection Systems," T. P. Malone, SAND880573, Sandia National Laboratories, 1988.
Munno-93 "Automatic Video Image Moving Target Detection for Wide Area Surveillance," C. J. Munno, Proc. IEEE Inter-national Carnahan Conference on Security Technology, 1993.

Pritchard-90 "An Infrared Imaging Area Sensor for Tactical and Physical Security Applications," D. A. Pritchard, Proc. IRIS Specialty Group on Passive Sensors, ERIM, Ann Arbor, MI, March 1990.

Pritchard-92 "Status of the Video Imaging System for Detection, Tracking and Assessment (VISDTA) Scanning Surveillance Sensor Program," D. A. Pritchard, Proc. IEEE International Carnahan Conference on Security Technology, 1992.

Pritchard-93 "Evaluation of Automatic Detection of Humans and Vehicles," D. A. Pritchard, R. F. Davis, and J. E. Simpson, Proc. IRIS Specialty Group on Targets, Backgrounds, and Discrimination, ERIM, Ann Arbor, MI, January 1993.

US Army-93 FLIR92 Thermal Imaging Systems Performance Model, U. S. Army Night Vision and Electronic Sensors Directorate, Document 5008993, Fort Belvoir, VA, 1993.

Vigil-92 "An Evaluation of Exterior Video Motion Detection Systems, Volume 1: Intrusion Detection Tests," J. T. Vigil, SAND92-0108, Sandia National Laboratories, 1992. 

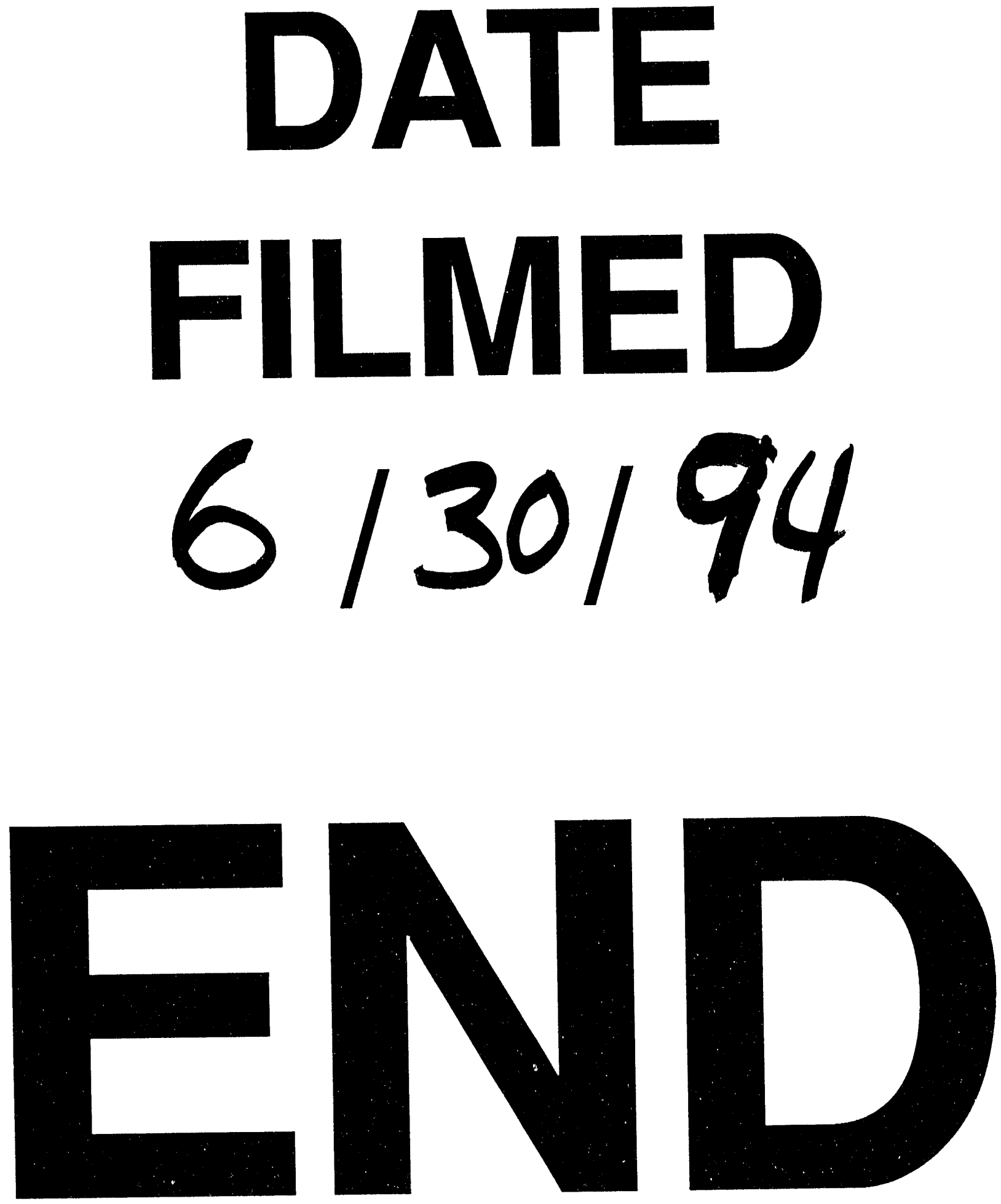


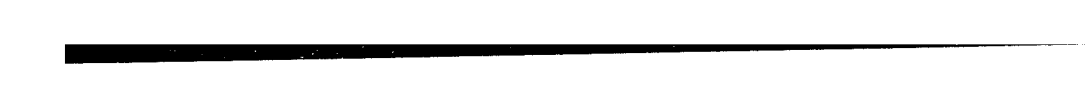

